\title{
Role of Sequential Treatment of Paracentesis Followed by Laser Peripheral Iridotomy in Acute Primary Angle Closure
}

\author{
Widya Artini, ${ }^{1,2}$ Tjahjono D Gondowiardjo, ${ }^{1,2}$ Edi S Afandi ${ }^{2}$ \\ ${ }^{\top}$ Department of Ophthalmology, Faculty of Medicine, Universitas Indonesia, and \\ ${ }^{2}$ Department of Ophthalmology, Cipto Mangunkusumo General Hospital, Jakarta, Indonesia
}

\begin{abstract}
Aim: To assess the role of sequential treatment of paracentesis followed by laser peripheral iridotomy in acute primary angle closure.

Methods: This was a prospective interventional study of acute primary angle closure in Indonesian patients. All eyes underwent paracentesis immediately after admission to reduce the intraocular pressure, followed by laser peripheral iridotomy on the following day to relieve pupillary blockade. A comprehensive eye examination was performed. The criterion for success was intraocular pressure $<21 \mathrm{~mm} \mathrm{Hg}$ with or without glaucoma medication. Risk factors assessed were age, duration of acute symptoms, presenting intraocular pressure, intraocular pressure response to paracentesis and to laser peripheral iridotomy, anterior chamber depth, and extent of peripheral anterior synechiae.

Results: Forty five eyes of 45 patients with acute primary angle closure were recruited, of whom 38 were women. The duration of symptoms ranged from 2 to 30 days (median, 14 days). The mean extent of peripheral anterior synechiae was 7.7 clock hours (SD, 3.1 clock hours) and the mean presenting intraocular pressure was $55.9 \mathrm{~mm} \mathrm{Hg}(\mathrm{SD}, 13.4 \mathrm{~mm} \mathrm{Hg}$; range, 30.0-78.0 $\mathrm{mm} \mathrm{Hg}$ ). After paracentesis, the mean intraocular pressure decreased to 27.0 (SD, $12.8 \mathrm{~mm} \mathrm{Hg}$; range, 15.0-54.0 $\mathrm{mm} \mathrm{Hg}$ ). After laser peripheral iridotomy, the mean intraocular pressure was $24.0 \mathrm{~mm} \mathrm{Hg}(\mathrm{SD}, 15.2 \mathrm{~mm} \mathrm{Hg}$; range 8.0-40.0 $\mathrm{mm} \mathrm{Hg}$ ). Success was achieved in 21 eyes and the variable risk factor influencing success was extent of peripheral anterior synechiae.
\end{abstract}

Conclusion: The success rate for sequential treatment was $47 \%$ of affected eyes and was determined mostly by the extent of peripheral anterior synechiae.

\footnotetext{
Key words: Glaucoma, angle closure, Intraocular pressure, Iridotomy, Lasers, Paracentesis, Peripheral anterior synechiae

Asian J Ophthalmol. 2011;13:3-8.
}

\section{Introduction}

A survey conducted by the Ministry of Health, Republic of Indonesia, in 1996 showed that the blindness rate in Indonesia was 1.5\%, and glaucoma emerged as the second leading cause of blindness $(0.2 \%)^{1}$

Acute primary angle closure (APAC) is caused by the closure of all of the trabecular meshwork due to peripheral iris bowing, which causes a sudden increase in intraocular pressure (IOP). Persistently high IOP tends to damage the retinal ganglion cells through necrosis-apoptosis and this may result in optic nerve

Correspondence: Dr Widya Artini, Department of Ophthalmology, Faculty of Medicine, Universitas Indonesia, Jl Kimia No 8, Jakarta Pusat 1350, Indonesia.

Tel: (62 21) 3193 4878;

E-mail: ikkesumantri@gmail.com atrophy. The disease is responsible for most bilateral glaucomarelated permanent blindness. ${ }^{2,3}$ It has been reported that the annual incidence of APAC in Asian populations is $0.3 \%$ to $1.6 \%$ higher than in Caucasian populations. ${ }^{2,3}$

Correct management of this disease is extremely important as blindness in the affected eye can be prevented. Long-term reduction of IOP by laser peripheral iridotomy (LPI) has been reported. ${ }^{4-6}$ This intervention has shown satisfactory results in Caucasians, but not in Asians due to the more severe inflammatory reaction and formation of peripheral anterior synechiae (PAS) in this population. ${ }^{5,6}$ Paracentesis for early management of APAC has shown satisfactory results. ${ }^{7}$ However, the definitive treatment remains as peripheral iridectomy to eliminate the pupillary block mechanism. ${ }^{3,8}$ There are insufficient data on the factors that influence the successful response to sequential intervention of paracentesis followed by LPI 
in Indonesian eyes with APAC. The objective of this study was to evaluate the treatment regimen of paracentesis followed by LPI for APAC in an Indonesian population and to determine the possible risk factors.

\section{Methods}

This study was a prospective cross-sectional study conducted at the Department of Ophthalmology, Cipto Mangunkusumo General Hospital, Jakarta, Indonesia. The authors obtained prior approval for the study protocol from the Ethics Committee of the Faculty of Medicine at the Universitas Indonesia. The study was conducted according to the principles of the Declaration of Helsinki, and written informed consent was obtained from all patients before recruitment.

\section{Patients}

Patients enrolled in the study were indigenous Indonesian nationals presenting with symptoms of APAC for less than 1 month, who were recruited consecutively between November 2005 and December 2007. The diagnostic criteria used for APAC were presence of at least 2 symptoms of eye pain, headache, blurred vision, or vomiting; presence of conjunctival congestion, mid-dilated unreactive pupil, and corneal oedema; $\geq 270^{\circ}$ of anterior chamber angle closure on gonioscopy examination; and IOP $>40 \mathrm{~mm} \mathrm{Hg}$ by Goldmann applanation tonometry. ${ }^{8}$ Patients were excluded if they had undergone any prior laser or surgical procedure; been given more than 3 types of glaucoma medication prior to admission; a reported history or observable signs of trauma to the eye; unwillingness to provide consent; or inability to return for scheduled visits.

\section{Design}

All patients underwent an initial eye examination at admission and up to day 14 consisting of IOP measurement by Goldmann applanation tonometry (mean of 3 readings), best-corrected visual acuity (BCVA) by Snellen chart, which was subsequently converted to LogMAR, slit-lamp examination, optic nerve assessment by noncontact lens, static and dynamic gonioscopy with assessment of PAS extent and angle grading (using the modified Shaffer grading). Gonioscopy was performed by one author and IOP measurement was done by one author.

The extent of PAS in clock hours was graded by using a pie diagram up to day 14 after LPI. The diagram was divided into 4 quadrants, and each quadrant was divided into 3 radial sections, providing 12 radial sections, which were labelled 0 to 4,5 to 8 , and 9 to 12. Immersion biometry was done at admission by a senior technician. Up to day 45 (range, day 30 to day 45 ), visual field examinations were performed using static automated white- on-white threshold perimetry (program 24-2, SITA standard). All clinical data were documented on standard forms. Two glaucoma specialists performed this comprehensive ophthalmic examination.

Paracentesis was done at presentation (day 1) for each eye with APAC as follows. With the patient in the supine position under the operating microscope, the affected eye was rinsed with povidoneiodine $20 \%$, then paracentesis was performed using a $30 \mathrm{G}$ needle. The needle was inserted from the limbus into the anterior chamber parallel to the iris. Approximately $150 \mu \mathrm{L}$ of aqueous humour was taken, and ciprofloxacin hydrochloride eye drops were given for 5 days. ${ }^{7}$ Each aqueous humour sample was placed into an Eppendorf tube and frozen at $-80^{\circ} \mathrm{C}$ until cytokine measurement was performed. The level of transforming growth factor- $\beta 2$ in the aqueous humour was measured by enzyme-linked immunosorbent assay by the Quantikine procedure. The affected eyes were given prednisolone acetate and ciprofloxacin hydrochloride eye drops 4 times daily without any glaucoma medication for 7 days.

Sequential LPI was performed on all eyes the day after paracentesis. All paracentesis and laser procedures were performed by 2 senior glaucoma specialists in the outpatients clinic. The affected eye was prepared half an hour before LPI by administering pilocarpine $2 \%$ and brimonidine eye drops. Iridotomy was performed by using an Abraham lens and applying a diode-pump frequency-doubled solid-state $532 \mathrm{~nm}$ laser (Visulas Combi; Carl Zeiss Meditec AG, Jena, Germany) with power settings of 700 to $900 \mathrm{~mW}$ for $50 \mu \mathrm{m}$ for 0.05 to 0.10 seconds This was followed by Nd-YAG laser (Visulas Combi; Carl Zeiss Meditec AG) with a 1.5 to $2.0 \mathrm{~mJ}$ power setting. ${ }^{9}$ The laser beam was aimed at the 10 o'clock or the 20 'clock position, two-thirds of the way from the pupil to produce an iridotomy hole of at least $200 \mu \mathrm{m}$. After LPI, prednisolone acetate and ciprofloxacin hydrochloride eye drops were administered for 5 days. The IOP was measured at 1 hour, 7 days, and 14 days after LPI. During follow-up, if the IOP was $>21 \mathrm{~mm} \mathrm{Hg}$, timolol $0.5 \%$ with or without brimonidine twice daily was given. The success of this sequential treatment was determined by achievement of an IOP $<21 \mathrm{~mm} \mathrm{Hg}$ with or without medical therapy for up to 2 weeks. Failure was determined by an IOP $>21 \mathrm{~mm} \mathrm{Hg}$.

Risk factors of age, duration of symptoms, presenting IOP, IOP response to paracentesis ( 6 hours after paracentesis), IOP response to LPI (up to 2 weeks after LPI), anterior chamber depth (during admission), and PAS extent in clock hours (up to 2 weeks after LPI) were assessed.

\section{Statistical Analysis}

Statistical analysis was performed using the Statistical Package for the Social Sciences 15.0 for Windows (SPSS Inc, Chicago, 
Illinois, USA). A sample size was calculated using a significance level of 0.05 with a power of 0.8 . Continuous variables were expressed as mean (standard deviation) or median (minimummaximum). Categorical variables were expressed as frequency and percentage. The Wilcoxon test (non-normal distribution) was used to compare the IOP before and after paracentesis and LPI. Bivariate analysis was calculated by using chi-squared or Fisher exact test to determine any correlation of each variable of possible risk such as sex, age, duration of acute symptoms, presenting IOP, response to paracentesis, anterior chamber depth, and PAS extent to predict the likely successful response. Of these, the variables that had the $p$ value $<0.25$ of bivariate analysis were further evaluated using logistic regression models to determine the adjusted relative risk (RR) and 95\% confidence interval $(\mathrm{Cl})$ and to determine the probability of a successful outcome. A p value of $<0.05$ was considered to be statistically significant. The discrimination and calibration value of logistic regression model were considered good if the area under the curve resulting from the receiver operating characteristic curve was $\geq 80 \%$ and the $\mathrm{p}$ value of the Hosmer-Lemeshow test was $>0.05$, respectively.

\section{Results}

\section{Demographic and Clinical Data}

Forty five eyes of 45 patients with APAC who underwent paracentesis followed by LPI were recruited into this study. Thirty eight patients $(84.4 \%)$ were women and $7(15.6 \%)$ were men. The mean age was 54.6 years (SD, 1.6 years; range, $40-77$ years). The mean IOP at presentation was $55.9 \mathrm{~mm} \mathrm{Hg} \mathrm{(SD,} 13.4 \mathrm{~mm} \mathrm{Hg}$; range, $30.0-78.0 \mathrm{~mm} \mathrm{Hg}$ ). The mean duration of acute symptoms was 13.2 days (SD, 7.4 days; range, 2-30 days) and the mean extent of PAS in clock hours was 7.7 (SD, 3.1; range, 4.0-12.0). The mean anterior chamber depth was $2.5 \mathrm{~mm}(\mathrm{SD}, 0.2 \mathrm{~mm}$; range, 2.1$2.9 \mathrm{~mm}$ ) and the mean axial length was $22.2 \mathrm{~mm}(\mathrm{SD}, 0.7 \mathrm{~mm}$; range 23.1-21.2 $\mathrm{mm}$ ) [Table 1].

\section{Clinical Outcomes}

Table 2 shows the response of eyes with APAC to paracentesis and LPI. The mean IOP decreased to $27.0 \mathrm{~mm} \mathrm{Hg}$ (SD, $12.8 \mathrm{~mm} \mathrm{Hg}$; range, $15.0-54.0 \mathrm{~mm} \mathrm{Hg}$ ) after paracentesis and to 24.0 (SD, $15.2 \mathrm{~mm} \mathrm{Hg}$; range 8.0-40.0 $\mathrm{mm} \mathrm{Hg}$ ) after LPI. The decrease in IOP after paracentesis was nearly $49 \%$ of the presenting IOP $(p \leq 0.001)$ [Figure 1]. The success rate after LPI was $47 \%$ (21 eyes) [Table 3].

Table 4 shows the clinical appearance of APAC in relation to the duration of acute symptoms that characteristically influence the clinical outcome. The mean cup-disc ratio was 0.8 (SD, 0.1), the mean visual acuity was 0.7 LogMAR (SD, 0.4 LogMAR) and
Table 1. Demographic data of patients with acute primary angle closure.

\begin{tabular}{|lc|}
\hline Characteristic & Mean (SD) \\
\hline Sex, Number (\%) & \\
$\quad$ Female & $38(84.4)$ \\
Male & $7(15.6)$ \\
Age (years) & $54.6(1.6)$ \\
PAS (clock hours) & $7.7(3.1)$ \\
Duration of acute symptoms (days) & $13.2(7.4)$ \\
Presenting IOP (mm Hg) & $55.9(13.4)$ \\
Anterior chamber depth (mm) & $2.5(0.2)$ \\
Axial length (mm) & $22.2(0.7)$ \\
Lens thickness (mm) & $4.7(0.6)$ \\
Cup-disc ratio & $0.64(0.2)$ \\
Visual field (mean deviation) & $-14.7(13.5)$ \\
Visual acuity & $1.2(0.7)$ \\
TGF- $\beta 2$ (pg/mL) & $2007.7(827.2)$ \\
\hline
\end{tabular}

Abbreviation: $\mathrm{IOP}=$ intraocular pressure; $\mathrm{PAS}=$ peripheral anterior synechiae; $\mathrm{TGF}=$ transforming growth factor.

Table 2. Intraocular pressure response of eyes with acute primary angle closure to paracentesis and laser peripheral iridotomy.

\begin{tabular}{|lcr|}
\hline Intervention & $\begin{array}{c}\text { Intraocular pressure (SD) } \\
\text { [mm Hg] }\end{array}$ & $\begin{array}{c}\text { Range } \\
\text { (mm Hg) }\end{array}$ \\
\hline Paracentesis & $27(12.8)$ & $15.0-54.0$ \\
Laser peripheral iridotomy & $24(15.2)$ & $8.0-40.0$ \\
\hline
\end{tabular}

Figure 1. Response to paracentesis in eyes with acute primary angle closure. ${ }^{*} \mathrm{p}<0.001$, Wilcoxon test.

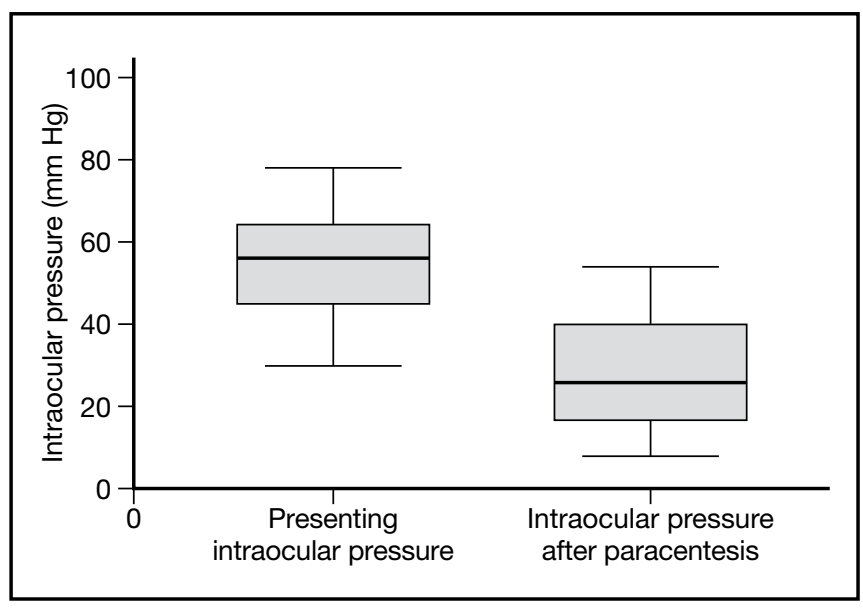

Table 3. Success rate of eyes with acute primary angle closure after sequential treatment of paracentesis followed by laser peripheral iridotomy.

\begin{tabular}{|lc|}
\hline Outcome & Number (\%) \\
\hline Success & $21(46.7)$ \\
Failure & $24(53.3)$ \\
\hline
\end{tabular}

the mean visual field was -24.1 (SD, 11.9) in 26 eyes with a duration of acute symptoms of $>7$ days.

Table 5 shows the bivariate analysis of the relationship of possible risk factors and the success of paracentesis and LPI to determine possible causes of failure. Table 6 shows the IOP response to paracentesis by multivariate analysis ( $R R, 8.03)$; eyes 
Table 4. Duration of symptoms and the relationship with cup-disc ratio, visual acuity, and visual field.

\begin{tabular}{|lcccc|}
\hline Duration of symptoms (days) & Number of patients & $\begin{array}{c}\text { Cup-disc ratio } \\
\text { Mean (SD) }\end{array}$ & $\begin{array}{c}\text { Visual acuity (LogMAR) } \\
\text { Mean (SD) }\end{array}$ & $\begin{array}{c}\text { Visual field (mean deviation) } \\
\text { Mean (SD) }\end{array}$ \\
\hline$\leq 7$ & 19 & $0.5(0.2)$ & $0.1(0.1)$ & $-7.7(8.8)$ \\
$>7$ & 26 & $0.8(0.1)$ & $0.7(0.4)$ & $-24.1(11.9)$ \\
Range & & $0.2-1.0$ & $0-2.0$ & $-40.0--0.6$ \\
\hline
\end{tabular}

Table 5. Bivariate analysis of the relationship between risk factors and the success of sequential paracentesis and laser peripheral iridotomy in eyes with acute primary angle closure.

\begin{tabular}{|c|c|c|c|c|c|}
\hline Risk factor & $\begin{array}{l}\text { Successful } \\
\text { intervention }\end{array}$ & $\begin{array}{c}\text { Failed } \\
\text { intervention }\end{array}$ & p Value & Relative risk & $\begin{array}{l}95 \% \text { confidence } \\
\text { interval }\end{array}$ \\
\hline \multicolumn{6}{|l|}{ Sex } \\
\hline Male & 2 & 5 & & & \\
\hline Female & 19 & 19 & 0.8260 & 1.105 & $0.44-2.77$ \\
\hline \multicolumn{6}{|l|}{ Age (years) } \\
\hline$\leq 60$ & 15 & 21 & 0.1760 & 0.630 & $0.34-1.14$ \\
\hline$>60$ & 6 & 3 & & & \\
\hline \multicolumn{6}{|c|}{ Duration of symptoms (days) } \\
\hline$\leq 7$ & 13 & 6 & 0.0170 & 2.220 & $1.16-4.27$ \\
\hline$>7$ & 8 & 18 & & & \\
\hline \multicolumn{6}{|c|}{ Presenting intraocular pressure $(\mathrm{mm} \mathrm{Hg})$} \\
\hline$\leq 50$ & 12 & 7 & 0.0750 & 1.825 & $0.97-3.43$ \\
\hline$>50$ & 9 & 17 & & & \\
\hline \multicolumn{6}{|c|}{ Paracentesis response $(\mathrm{mm} \mathrm{Hg})$} \\
\hline Good $\leq 22$ & 15 & 4 & $<0.0001$ & 3.420 & $1.63-7.17$ \\
\hline Poor $>22$ & 6 & 20 & & & \\
\hline \multicolumn{6}{|c|}{ Anterior chamber depth $(\mathrm{mm})$} \\
\hline$\leq 1.4$ & 8 & 22 & $<0.0001$ & 0.276 & $0.14-0.54$ \\
\hline$>1.4$ & 13 & 2 & & 3.250 & $1.74-6.08$ \\
\hline \multicolumn{6}{|c|}{ PAS (clock hours) } \\
\hline $0-4$ & 12 & 3 & $<0.0001$ & 4.8 & $1.65-13.90$ \\
\hline $5-8$ & 6 & 6 & 0.0510 & 3.0 & $0.92-5.04$ \\
\hline $9-12$ & 3 & 15 & & & \\
\hline
\end{tabular}

Abbreviation: PAS = peripheral anterior synechiae.

with a lesser extent of PAS (0-4) had an RR of 9.45 compared with an RR of 2.41 for eyes with greater PAS (5-8).

Clinical variables were calculated by the equation of probability formula according to IOP response to paracentesis (RR, 8.03) and extent of PAS (RR, 9.45). For IOPs $<22 \mathrm{~mm} \mathrm{Hg}$ and extent of PAS $<4$, paracentesis and LPI would have a success rate probability of $95 \%$ (Table 7), and for IOPs $>22 \mathrm{~mm} \mathrm{Hg}$ and extent of PAS $>9$, the success rate probability would be $5 \%$ (sensitivity, 90.5 ; specificity, 70.8; accuracy value, 8; cut-off point, 0.5 ).

Figure 2 shows the receiver operating characteristic value of $0.89(p=0.917$; discrimination value, $89.2 \%)$.

\section{Discussion}

In APAC, pupillary block leads to higher pressure in the posterior chamber than in the anterior chamber as a result of increases in iris convexity that brings the peripheral iris into contact with the anterior wall of the angle, thereby closing the angle. The longer the duration of acute symptoms, the more extensive will be the PAS. In addition, the persisting high IOP would be more difficult to manage. These conditions affect the optic nerve, with the retinal ganglion cells and visual fields being severely damaged, ${ }^{10.11}$ as happened to the patients in this study.

The management of APAC is crucial. Prompt treatment is associated with a good prognosis. Treating an acute attack early will minimize damage to the optic nerve, retinal ganglion cells, and trabecular meshwork. Early treatment also reduces the formation of PAS. At present, the definitive treatment remains LPI in both the involved and fellow eyes. ${ }^{3.4 .6}$

Even though paracentesis was performed immediately after diagnosis to reduce the IOP quickly, only 19 eyes had a good outcome. However, this intervention was sufficiently effective to clear the cornea, making it possible for LPI to be performed. Paracentesis as an initial intervention for eyes with APAC has been supported by Lam et al, ${ }^{7}$ who demonstrated that this intervention decreases the IOP immediately with $100 \%$ success. However, all of the patients in Lam et al's study presented within 24 hours of the start of the attack, ${ }^{7}$ which is significantly earlier than our patients, who mostly presented with a duration of attack longer than 7 days.

The most notable aspect of this study was that the success rate for paracentesis followed by LPI was only $47 \%$ for up to 2 weeks of 
Table 6. Multivariate analysis of the relationship between risk factors and the success of sequential paracentesis and laser peripheral iridotomy in eyes with acute primary angle closure.

\begin{tabular}{|c|c|c|c|c|c|}
\hline \multirow[t]{2}{*}{ Risk factor } & \multicolumn{2}{|c|}{ Bivariate analysis } & \multicolumn{3}{|c|}{ Multivariate analysis } \\
\hline & Relative risk & p Value & Relative risk & 95\% confidence interval & p Value \\
\hline IOP response to paracentesis & 3.42 & $<0.0001$ & 8.03 & $2.30-13.39$ & 0.003 \\
\hline PAS 0-4 & 4.80 & $<0.0001$ & 9.45 & $2.45-14.30$ & 0.004 \\
\hline PAS 5-8 & 3.00 & 0.0510 & 2.41 & $0.37-8.95$ & 0.339 \\
\hline
\end{tabular}

Abbreviation: IOP = intraocular pressure; PAS = peripheral anterior synechiae.

Table 7. Results of the prediction model for success of paracentesis and laser peripheral iridotomy.

\begin{tabular}{|lcc|}
\hline $\begin{array}{l}\text { Intraocular pressure } \\
(\mathbf{m m ~ H g})\end{array}$ & $\begin{array}{c}\text { Peripheral anterior synechiae } \\
\text { (clock hours) }\end{array}$ & $\begin{array}{c}\text { Success of paracentesis and } \\
\text { laser peripheral iridotomy }\end{array}$ \\
\hline$\leq 22$ & $\leq 4$ & 0.95 \\
$\leq 22$ & $5-8$ & 0.73 \\
$\leq 22$ & $9-12$ & 0.33 \\
$>22$ & $\leq 4$ & 0.67 \\
$>22$ & $5-8$ & 0.23 \\
$>22$ & $9-12$ & 0.05 \\
\hline
\end{tabular}

Figure 2. Receiver operating characteristic curve.

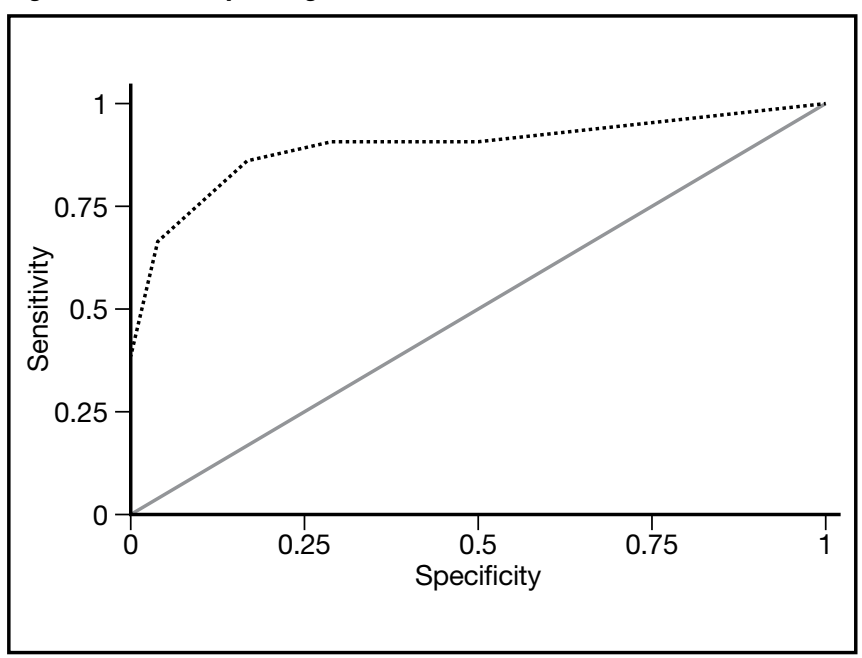

observation. This was lower than any other reported study. ${ }^{6.12-17}$ It is possible that the affected eyes in this study demonstrated severe clinical symptoms as well as severe inflammation, in addition to the long duration of acute symptoms. These factors seem to be the most influential for treatment failure. ${ }^{18,19}$ Persistent inflammation due to high cytokine levels is likely to increase PAS formation, or there may be other factors involved such as lens dimension, iris thickness, severe trabecular meshwork damage, or choroidal effusion. ${ }^{20.21}$

When risk factors related to the success rate of this sequential intervention were evaluated in this study population, the IOP response to paracentesis and the extent of PAS were the two most influential factors. If the response to paracentesis was good and the extent of PAS was less than 4 clock hours, the probability of a successful response to LPI was as high as $95 \%$. However, if the response to paracentesis was not good and the extent of PAS was as much as 8 to 12 clock hours, the probability of success was only $5 \%$.

This study improves our understanding of the management of APAC in this community. If only LPI is done, patients must be followed up more closely. This raises questions as to what strategy should be used if a patient lives far from a well-equipped hospital or is economically disadvantaged. It may be that management of APAC in this population should be decided based on the extent of PAS. If the extent of PAS is $>270^{\circ}$, then a 2-stage intervention in one setting should be performed; paracentesis together with LPI plus laser gonioplasty or trabeculectomy primer with mitomycin C. However, this recommendation needs further study. If the extent of PAS is $<180^{\circ}$, treatment is likely to be more successful. As highlighted by Aung et al, ${ }^{22}$ trabeculectomy is not recommended as a first-line treatment in Asian patients who do not respond well to initial medical treatment for APAC. It is postulated that trabeculectomy will give poor results in an inflamed eye. ${ }^{22}$ Preliminary data on cataract extraction, goniosynechialysis, and iridoplasty indicate that these approaches may be effective for treating APAC that cannot be controlled medically after LPI has been performed. ${ }^{23-25}$

There are some limitations to this study. Determination of the extent of PAS was a subjective examination, which may have introduced bias. This was reduced by providing the information on extent of PAS as a pie chart, with one specialist performing the examination. Further, this study had a small number of patients, which might influence the risk factor calculation result. A larger number of participants would be preferable.

In this study, the success rate for sequential intervention of paracentesis followed by LPI was only $47 \%$ due to the long duration of acute symptoms, which increased the extent of PAS extent. If 
the IOP was $>21 \mathrm{~mm} \mathrm{Hg}$ after these interventions and the extent of PAS was less than 4 , the probability of success was $95 \%$.

\section{References}

1. National Eye Health Survey 1993-1996. Jakarta: Ministry of Health; 1998.

2. Congdon N, Wang F, Tielsch JM. Issues in the epidemiology and population-based screening of primary angle closure glaucoma. Surv Ophthalmol. 1992;36:411-23.

3. Stamper RL, Lieberman MF, Drako MV. Primary angle closure glaucoma. In: Stamper RL, Lieberman MF, Drake MV, editors. Becker-Shaffer's diagnosis and therapy of the glaucoma. 8th ed. St Louis: Mosby; 2009. p 188-207.

4. Ritch R, Lowe RF. Angle closure glaucoma: clinical types. In: Ritch $\mathrm{R}$, Shields MB, Krupin T, editors. The glaucomas. 2nd ed. St Louis: Mosby; 1996. p 821-40.

5. Rosman M, Aung T, Ang LP, Chew PT, Liebmann JM, Ritch R. Chronic angle-closure with glaucomatous damage, long-term clinical course in a North American population and comparison with an Asian population. Ophthalmology. 2002;109:2227-31

6. Aung T, Ang LP, Chan SP, Chew PT. Acute primary angle closure: long term intraocular pressure outcome in Asian eyes. Am J Ophthalmol. 2001;131:7-12.

7. Lam DS, Chua JK, Tham CC, Lai JS. Efficacy and safety of immediate anterior chamber paracentesis in the treatment of acute primary angle closure glaucoma. Ophthalmology. 2002;109:64-70.

8. Saw SM, Gazzard G, Friedman DS. Interventions for angle-closure glaucoma; evidence based update. Ophthalmology. 2003;110: 1869-79.

9. South East Asia Glaucoma Interest Group. Asia Pacific glaucoma guidelines. Singapore: SEAGIG; 2004.

10. Aung T, Looi A, Chew PT. The visual field following acute primary angle closure. Acta Ophthalmol Scand. 2001;79:298-300.

11. Gazzard G, Foster PJ, Devereux JG, et al. Intraocular pressure and visual field loss in primary angle closure and primary open angle glaucomas. Br J Ophthalmol. 2003;87:720-5.

12. Krupin T, Mitchell KB, Johnson MF, Becker B. The long term effect of iridectomy for primary acute angle closure glaucoma. Am J Ophthalmol. 1978;88:506-9.

13. Playfair J, Watson PG. Management of acute primary angle closure glaucoma: a long term follow up of the results of peripheral iridectomy used as an initial procedure. $\mathrm{Br} \mathrm{J}$ Ophthalmol. 1979;63:17-22.

14. Quigley HA. Long term follow up of laser iridotomy. Ophthalmology. 1981;88:218-24.

15. Fu ER, Tan BB, Seah LL. The role of laser iridotomy in acute primary angle closure glaucoma in oriental population. Ophthalmol Ind. 1993;14:29-32.

16. Fleck BW, Wright E, Fairley EA. A randomised prospective comparison of operative peripheral iridectomy and Nd:YAG laser iridotomy treatment of acute angle closure glaucoma: 3 year visual acuity and intraocular pressure control outcome. $\mathrm{Br} \mathrm{J}$ Ophthalmol. 1997;81:884-8.

17. Buckley SA, Reeves B, Burdon M, et al. Acute angle closure glaucoma: relative failure of YAG iridotomy in affected eyes and factors influencing outcome. Br J Ophthalmol. 1994;78:529-33.

18. Tripathi RC, Li J, Chan WFA, Tripathi BJ. Aqueous humor in glaucomatous eyes contains an increased level of TGFB2. Exp Eye Res. 1994;39:723-8.

19. Inatani $\mathrm{M}$, Tanihara $\mathrm{H}$, Katsuta $\mathrm{H}$, Honjo $\mathrm{M}$, Kido $\mathrm{N}$. Transforming growth factor Beta 2 levels in aqueous humor of glaucomatous eyes. Graefes Arch Clin Exp Ophthalmol. 2001;239:109-13.

20. Yang M, Aung T, Husain R, et al. Choroidal expansion as a mechanism for acute primary angle closure: an investigation into the change of biometric parameters in the first 2 weeks. $\mathrm{Br} \mathrm{J}$ Ophthalmol. 2005;89:288-90.

21. Tarongoy P, Ho CL, Walton DS. Angle-closure glaucoma: the role of the lens in the pathogenesis, prevention, and treatment. Surv Ophthalmol. 2009;54:211-25

22. Aung T, Tow SL, Yap EY, Chan SP, Seah SK. Trabeculectomy for acute primary angle closure. Ophthalmology. 2000;107:1298-302.

23. Lam DS, Lai JS, Tham CC. Immediate argon laser peripheral iridoplasty as treatment for acute attack of primary angleclosure glaucoma: a preliminary study. Ophthalmology. 1998;105:2231-6.

24. Teekhasaenee C, Ritch R. Combined phacoemulsification and goniosynechialysis for uncontrolled chronic angle-closure glaucoma after acute angle-closure glaucoma. Ophthalmology. 1999;106: 669-74.

25. Jacobi PC, Dietlein TS, Luke C, Engles B. Phacoemulsification and intraocular lens implantation for acute angle-closure glaucoma Ophthalmology. 2002;109:1597-603. 